\title{
Final crop of 1983 Nobel awards
}

\section{The Nobel committees have awarded this year's physics prize to Professors W. A. Fowler (Caltech) and S. Chandrasekhar (Chicago) and the chemistry prize to Professor Henry Taube (Stanford).}

THE 1983 physics prize is shared between Dr S. Chandrasekhar (see following page) and Professor William A. Fowler, until his recent retirement professor of physics at the California Institute of Technology, Pasadena. Fowler's share of the prize is awarded for his experimental and theoretical studies of nuclear reactions relevant to the theory of nucleogenesis, one of the outstanding landmarks of the 1950s.

By background a nuclear physicist, Fowler has since the Second World War been the one chiefly responsible for making Caltech a prolific source of fruitful applications of nuclear physics in other fields, many with an astrophysical or cosmological bearing. His distinctive contribution to the successful solution in 1957 of the problem of nucleogenesis stemmed from his careful experimental measurements of many of the cross-sections of nuclear reactions thought to be crucial for energy production and the synthesis of heavy elements in stars. This problem was the central theme of Fowler's long spell as director of the Kellogg Laboratory at Caltech, in which role his physical intuition and enthusiasm is by common consent acknowledged to have been a powerful stimulus for a generation of students and graduate students. His geniality is legendary.

Fowler's best-known contribution to the understanding of the origin of the elements was by means of his collaboration with $\mathrm{Dr}$ (now Sir) Fred Hoyle (now visiting professor at University College, Cardiff), Dr Geoffrey R. Burbidge (director of the Kitt Peak Observatory, Arizona) and Dr Margaret Burbidge (University of California, San Diego). The first complete statement of their solution, a monumental paper now generally known as "BBF\&H", appeared in 1957 (Rev. mod. Phys. 29, 547-650; 1957).

One of the obvious difficulties for the Nobel committees must have been that the prize by-laws prevent the splitting of a prize between more than three people. Another is that similar proposals to those in BBF\&H were put forward at about the same time in a report by A.G.W. Cameron circulated by the Chalk River Laboratory of Atomic Energy of Canada Limited (CRL-41).

The distinctive feature of BBF\&H is that it is, above all, a comprehensive paper. The synthesis of helium from hydrogen by the intervention of $\mathrm{C}, \mathrm{N}$ and $\mathrm{O}$ nuclei as catalysts had been known from Bethe's work in 1938. Hoyle had argued in 1946 that the synthesis of nuclei of elements in the iron group could be accounted for by a kind of thermodynamic equilibrium at stellar temperatures, pointing to the potential importance of neutron addition. Most theories seeking to account for the occurrence of heavy elements, such as those of Teller and Gamow, had however supposed that the process responsible would have been a feature of the primordial unexpanded universe (although BBF\&H acknowledges that theories due to Klein and, separately, Beskow and Treffenberg in 1947 had been compatible with nucleogenesis within stars, the central feature of the new proposal).

The commanding thesis of $\mathrm{BBF} \& \mathrm{H}$ is its convincing demonstration that nucleogenesis in stars is a sufficient explanation of observed nuclear abundances, with the important astrophysical consequence that a substantial number of existing stars must have been formed from material generated in stars whose evolution had been completed.

Technically, BBF\&H was most successful in accounting for the formation of those isotopes heavier than ${ }^{16} \mathrm{O}$ which are not also constituted from alpha-particles

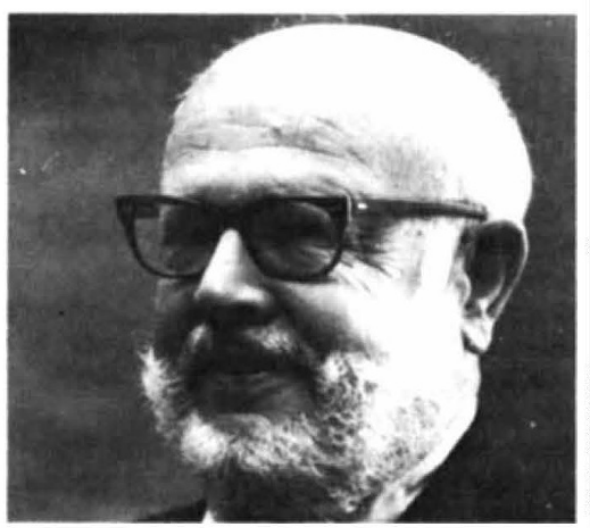

W.A. Fowler

by the addition of neutrons to lighter nuclei. Two distinct processes were suggested - the $s$ (for slow) process, by means of which heavy isotopes newly formed by neutron addition usually decay (by betadecay) before the addition of another neutron, and the $r$ (for rapid) process in which the neutron flux would have been sufficiently great to have generated nuclei heavier than bismuth.

One of the essential steps in the argument of BBF\&H was Fowler's confirmation, by means of experiments at Caltech, of an earlier prediction by Hoyle that there should be an excited state of the ${ }^{12} \mathrm{C}$ nucleus at an energy of about 7.6 MeV. Hoyle's prediction was derived from the assumption that the beginning of the red giant phase of the evolution of a star is marked by the onset of helium burning, in turn requiring a rate for the reaction between ${ }^{8} \mathrm{Be}$ and ${ }^{4} \mathrm{He}$ so great that it could be most simply accounted for by a near-coincidence ("resonance") of the combined energy of the two component nuclei with that of an excited state of ${ }^{12} \mathrm{C}$.

The theory of nucleogenesis of BBF\&H also stands out for the convincing identification that it first provided of realistic nuclear reactions that might serve as neutron sources in stars towards the end of the red giant phase. Fowler seems to have been chiefly responsible for drawing attention both to mechanisms by which oddmass isotopes such as ${ }^{21} \mathrm{Ne}$ may be formed (by proton reactions) and then serve as neutron sources (by interaction with ${ }^{4} \mathrm{He}$ nuclei). It seems, however, to have been Hoyle who first recognized the usefulness of the data on neutron UN conference on the Peacefire Uses of Atomic Energy in August 1955. The Burbidges, among other things, were able to contribute data on their observations of stars containing heavy elements in their envelopes.

BBF\&H is a landmark in the development of astrophysics for several reasons. The provision of a coherent explanation of nucleogenesis is one. Second, it settled once and for all an issue that had still be in doubt in the previous decade - the direction of stellar evolution along the Herzsprung-Russell diagram. Finally, it provided the objective basis for the calculations of the internal constitutions of stars that have since been carried out (crucial, for example, in the understanding of the place of globular clusters in the evolution of galaxies) as well as for the general understanding that the end-point of the evolution of a reasonably massive star is a supernova explosion.

The work described in BBF\&H was begun at Cambridge (England) during Fowler's tenure of a Fulbright professorship in 1954-55. The original version of the paper was rejected by the first US journal to which it was sent, but snapped up by $\mathrm{Dr}$ Lewis Branscomb, then editor of Reviews of Modern Physics. The final version of the paper was worked through by Hoyle and Fowler in 1957 when both were attending a meeting of the Pontifical Academy of Sciences at the Vatican.

John Maddox 